\title{
Producción de conocimiento para las políticas públicas en el nivel subnacional: la experiencia del Ministerio de Economía de la provincia de Buenos Aires (1987-2007) 1
}

\author{
Knowledge production for public policies at the subnational level: the experience of the \\ Ministry of Economy of the province of Buenos Aires (1987-2007)
}

\author{
Elsa Pereyra \\ Instituto del Conurbano - Universidad Nacional de General Sarmiento, \\ Escuela de Politica y Gobierno -Universidad Nacional de San Martín, \\ Argentina epereyra@ungs.edu.ar
}

\begin{abstract}
Resumen:
En este artículo analizo las estrategias que el equipo económico de la Provincia de Buenos Aires desarrolló entre 1987 y 2007, con el propósito de formar cuadros técnicos, producir conocimiento para las políticas, comunicar sus acciones e intervenir en los debates sobre política económica nacional y provincial. Estos desarrollos fueron parte de un movimiento más general que protagonizaron los economistas platenses, que excedió al Ministerio de Economía provincial y que supuso un contrapunto con el liderado por los centros de producción del pensamiento económico dominante de la época. La masa crítica de cuadros políticos y técnicos producida por la carrera de economía de la UNLP y la renovación peronista de mediados de la década de 1980, cuajó en grupos de investigación y análisis que generaron usinas locales cuyas perspectivas eran alternativas a ese pensamiento económico liderado por los principales thinktanks formados en la Argentina desde mediados de la década de 1970.
\end{abstract}

Palabras Clave: Saber especializado, Economistas, Nivel subnacional, Provincia de Buenos Aires.

\section{Abstract:}

In this paper, I analyze the strategies developed by the economic team of the Province of Buenos Aires between 1987 and 2007 in order to form technical cadres, obtain knowledge for the elaboration of policies, communicate their actions and participate $\mathrm{i}$ $\mathrm{n}$ debates on national and provincial economic policy. These developments were part of a more general movement carried out by local economists of La Plata, which exceeded the provincial Ministry of Economy and which differed from the dominant economic theoretical framework of the time, spearheaded by production centers. The critical mass of political and technical cadres produced by the UNLP economics career and the Peronist renewal movement of the mid-1980s came together in research and analysis groups that generated local power plants, with alternative perspectives to that economic thought led by the main think tanks formed in Argentina since the mid-1970s.

KEYWORDs: Specialized knowledge, Economists, Subnational level, Province of Buenos Aires.

Las transformaciones producidas en el capitalismo desde el último cuarto del siglo pasado fueron acompañadas por un movimiento en el mundo de las ideas que vino a fortalecerlas por medio de su explicación y justificación, en una dinámica cuya abigarrada interpenetración hace difícil comprender unas sin el otro. En este marco, el saber especializado en economía se convirtió en un insumo crítico tanto para impulsar la acumulación como para compensar los desajustes provocados por ese mismo proceso: facilitó la redefinición de los contenidos y dirección de la dominación así como de las formas de su legitimación, revistiendo de neutralidad técnica intervenciones profundamente políticas para la producción y reproducción del sistema. ${ }^{2}$

En la experiencia argentina -así como en la de numerosos países de América Latina y el resto del mundonada expresó mejor las batallas por las ideas que la proliferación de los denominados thinktanks. Estas "usinas" organizadas en centros y fundaciones para la investigación y el análisis de la economía y la elaboración 
de recomendaciones de política, financiadas con los aportes de empresas o instituciones externas como las Fundaciones Ford y Rockefeller, se desarrollaron a partir de grupos de economistas con estudios de posgrado en el exterior, principalmente en universidades norteamericanas. ${ }^{3}$ Este predominio favoreció la internacionalización del saber económico sobre la base de la matriz neoclásica y sus variantes (teorías de las expectativas racionales, de los costos de transacción, etc.), desplazando teorías rivales en la explicación de lo económico y sus fundamentos.

La unificación de las formas de intervención y regulación de la economía se tradujo en un isomorfismo institucional que operó sobre la base de convicciones firmes en torno a la virtud de las medidas adoptadas en algunos casos, mientras que en otros tuvo un carácter instrumental (Fourcade-Gourinchas y Babb, 2002). El estado y sus oficinas perdieron el monopolio de las capacidades cognitivas, abriéndose el espacio para la construcción de una "racionalidad para-estatal" (Heredia, 2004) que tuvo como protagonistas a estas organizaciones.

Si la dinámica e implicancias de estos desarrollos han sido bien estudiadas en el nivel nacional, poco sabemos sobre su expresión en el nivel subnacional. ${ }^{4}$ La concentración de activos de intelligentsia técnica en la ciudad de Buenos Aires -sede del gobierno nacional que extiende su inevitable influencia al conjunto del paísasí como el énfasis -al menos en el campo de la política económica- en los fenómenos y comportamientos de carácter más agregado, han contribuido a privilegiar esta escala del análisis, y al mismo tiempo opacar lo que pudiera ocurrir en provincias y municipios, dando por sentado, las más de las veces, una recepción pasiva de tales dinámicas, implicancias e influencias.

En este trabajo me propongo trasladar el análisis de tales procesos y dinámicas al nivel subnacional, específicamente la provincia de Buenos Aires. ¿Es posible identificar en ella iniciativas y desarrollos en el campo de la producción de información y análisis económico con el objeto de influir sobre el rumbo de las políticas similares a los que se asocian con la escala nacional?; ¡o bien se produjo un consumo pasivo de las iniciativas y aportes que lideraron los poderosos thinktanks que desde el centro proyectaron su influjo al conjunto del país? Estas preguntas conducen a revisar la experiencia bonaerense que tuvo como protagonistas a los economistas platenses, que se tradujo en la creación de centros de producción y análisis económico vinculados con el Ministerio de Economía provincial, y cuyos primeros antecedentes se remontan a mediados de la década de 1970.

Desde que el peronismo tomó las riendas de la provincia de Buenos Aires en 1987, el Ministerio de Economía se destacó por el hecho de que sus cuadros altos y medios eran en su abrumadora mayoría economistas que, además de contar con solvencia técnica, tuvieron militancia o pertenencia políticopartidaria, y construyeron un sentido de pertenencia, perspectivas y formas compartidas de abordar las cuestiones. Entre el año indicado anteriormente y 2007, los elencos de este Ministerio, liderados por quienes sucesivamente ocuparon el máximo cargo de la cartera -Jorge Remes Lenicov (1989-1997), Jorge Sarghini (1997-2003) y Gerardo Otero (2003-hasta marzo de 2007)- mostraron una inusual estabilidad, lo que permitió su configuración como un equipo, y la unidad y coherencia de sus acciones.

Entre ellas, ocupó un lugar preponderante el desarrollo de una serie de estrategias y dispositivos destinados a la formación de cuadros técnicos, la producción de conocimiento, la comunicación de las medidas y políticas, y la intervención en los debates sobre política económica. Con ello el equipo económico buscaba estimular el conocimiento y la reflexión sobre la provincia de Buenos Aires y su lugar en el contexto nacional, y constituirse en referente en el campo de las finanzas públicas y el federalismo fiscal.

En este trabajo analizo los canales por los que esos intereses se concretaron: la promoción y financiamiento de un posgrado en finanzas públicas provinciales y municipales en la Facultad de Ciencias Económicas de la UNLP, y las publicaciones promovidas en el ámbito del Ministerio de Economía provincial, denominadas Cuadernos de Economía y Noticias de Economía. Asimismo, considero esos desarrollos como parte de un movimiento más general que tuvo como protagonistas a los economistas platenses, que excedió al Ministerio de Economía provincial y que supuso un contrapunto con el liderado por los centros de producción del 
pensamiento económico dominante de la época. La masa crítica de cuadros políticos y técnicos producida por la carrera de economía de la UNLP y la renovación peronista de mediados de la década de 1980, cuajaron en grupos de investigación y análisis que generaron usinas locales cuyas perspectivas eran alternativas a ese pensamiento económico liderado por los principales thinktanks formados en nuestro país desde mediados de la década de 1970 (FIEL, la Fundación Mediterránea y el CEMA).

En primer lugar analizo el contexto y las condiciones de creación y desarrollo de los grupos de investigación y análisis que dentro y fuera del Ministerio de Economía fueron alimentados y estimulados por integrantes del equipo económico provincial. Luego analizo las revistas Noticias de Economia y Cuadernos de Economía como productos del Ministerio de Economía provincial destinados a la comunicación de políticas y a la producción de conocimiento para las políticas, respectivamente. Finalmente reflexiono en torno al vínculo entre conocimiento y política, y los modos en que el equipo económico provincial construyó su autoridad política y técnica en determinadas cuestiones de política económica provincial -especialmente en el campo del federalismo fiscal y el problema de la coparticipación federal de impuestos- y se legitimó tanto en el ámbito provincial como en el nacional.

\section{IDEAS ADENTRo y AFUERA DEL Ministerio de EConomía PROVINCIAL: USINAS EN MARCHA}

Los economistas que forjaron el equipo del Ministerio de Economía provincial constituyeron un colectivo de entre treinta y cuarenta funcionarios, la gran mayoría de ellos hombres egresados de la Universidad Nacional de La Plata. Aun cuando entre sus filas se contaban algunas mujeres, esta precisión no es menor, ya que revela el sesgo de género que caracteriza a la profesión de economista así como a la ocupación de las posiciones superiores en la gestión pública. Esta experiencia singular tuvo como locus a la ciudad de La Plata, la capital de la Provincia de Buenos Aires marcada por su carácter administrativo y universitario. Sus figuras más prominentes se formaron en las escuelas de la militancia política en el seno del peronismo y de las aulas de la Facultad de Ciencias Económicas de la Universidad Nacional de La Plata. Este doble origen, reforzado por el sentido comunitario que la ciudad de La Plata contribuyó a desarrollar, constituyó un sello distintivo de este colectivo que lo diferencia de otros en los que la lealtad profesional se antepuso a la lealtad política, tal como lo sugieren Sergio Morresi y Gabriel Vommaro (2011).

Los pioneros del equipo económico provincial hicieron sus primeras armas en la militancia universitaria y tuvieron un paso por la gestión en el marco del segundo peronismo (1973-1976). En la evaluación de esa experiencia, el déficit en la capacidad analítica explicaba en gran medida el fracaso del gobierno. De ese balance surgió una perspectiva que promovía la formación de un colectivo reducido de cuadros dedicado al análisis del escenario nacional en sus diversas dimensiones, y la preparación para el gobierno sobre la base de estudios y proyecciones que previeran estrategias y medidas en diversas escalas temporales de la intervención. Ese colectivo debía reunir especialización y visión política, y erigirse en la dirección intelectual de los procesos políticos y económicos. A diferencia del Instituto Di Tella, modelo de referencia para esta perspectiva, el compromiso político constituía el reaseguro para que un colectivo semejante no incurriera en comportamientos "tecnocráticos".

Estas iniciativas tuvieron un primer ensayo durante el proceso de la renovación peronista, que en La Plata convocó,en el Centro de Estudios para la Renovación Justicialista (CEPARJ), a militantes con saber especializado para realizar análisis y diagnósticos de la situación provincial que contribuyeran a un plan de gobierno(Pereyra, 2016 y 2017). Pero fue a partir del acceso al Ministerio de Economía provincial desde 1987 que los economistas platenses contaron con condiciones y recursos para impulsar y sostener espacios en los que la capacidad analítica se desarrollara. La disposición de información y el trabajo sistemático sobre ella eran considerados por la mayoría de ellos como algo indispensable para la tarea de gobierno, y desde su punto de vista las oficinas provinciales -no sólo las del Ministerio de Economía provincial- tenían un déficit que era necesario resolver. 
Las estructuras de gestión del Ministerio de Economía parecían adecuadas para contener la actividad de análisis: las direcciones provinciales de estudios económicos y estudios fiscales estaban destinadas a tales propósitos. ${ }^{6}$ La primera de ellas apuntó a la realización de estudios de base con una perspectiva de mediano y largo plazo; en tanto que los trabajos de la segunda estaban sujetos a las necesidades de diseño de la política fiscal y tributaria. Los equipos de investigación de la Facultad de Ciencias Económicas de la UNLP, particularmente el dedicado al federalismo fiscal, constituían una fuente y una referencia para construir los argumentos de las políticas y medidas. Todo ello componía, en suma, una masa crítica de considerables proporciones a disposición del equipo de economía provincial.

Durante la década de 1990, esa masa crítica para el desarrollo de la capacidad analítica experimentó una expansión significativa, dando lugar a un conjunto de iniciativas que formaron un mosaico de centros de estudio, análisis y asistencia técnica de considerable vitalidad, y pivotearon en torno -aunque no exclusivamente- del Ministerio de Economía provincial. Entre los factores que explican tal despliegue quiero destacar tres: el interés por la formación de cuadros, las tensiones asociadas con las perspectivas de carreras individuales, y los conflictos frente a políticas nacionales y/o provinciales.

La formación de cuadros se definió tempranamente como un objetivo del equipo económico provincial. Involucró tanto la educación formal, expresada en la creación de un posgrado en finanzas públicas dentro de la Facultad de Ciencias Económicas de la UNLP, como la creación de estructuras dentro del Ministerio de Economía para el trabajo de investigación y análisis y la incorporación de jóvenes profesionales en las áreas de gestión. ${ }^{7}$ La primera ponía de manifiesto el interés de los líderes del equipo económico provincial en el vínculo académico y profesional con el Departamento de Economía de la Facultad de Ciencias Económicas de la UNLP. La segunda revelaba que las necesidades de desarrollo de capacidad analítica no terminaban de ser satisfechas por la institución académica con la que el Ministerio mantuvo relación tan cuidada: la generación de instancias propias de análisis e investigación aseguraba autonomía y criterios propios en la producción, y brindaba al equipo económico provincial mayores márgenes para la definición de los recursos a emplear.

La iniciativa de crear un posgrado en finanzas públicas subnacionales en el marco de la Facultad de Ciencias Económicas de la UNLP se verificó en una coyuntura favorecida por la presencia, en las instituciones involucradas, de figuras que ocupaban posiciones de relevancia y que por diversas razones estaban interesadas en la formación de profesionales especializados en dicho campo: Horacio Pereyra en el Consejo Federal de Inversiones (CFI); Alberto Porto en la Facultad de Ciencias Económicas de la UNLP, y el ya mencionado Remes Lenicov, Ministro de Economía de la Provincia de Buenos Aires. Los tres se conocían por haber compartido un espacio: la Facultad de Ciencias Económicas de la UNLP. ${ }^{8}$ Cada uno de ellos aportaba el knowhow y los propósitos de las instituciones que representaban. El CFI buscaba promover la formación de cuadros técnicos en las provincias en campos y temas que favorecieran el desarrollo de los estados subnacionales; el "DepEco" - tal como denominaban al Departamento de Economía de la UNLP los economistas platenses- aportaba su staff de profesores confiriendo prestigio académico al posgrado, y avanzaba en el desarrollo de la oferta académica contando con el financiamiento que le brindaban el CFI y el Ministerio de Economía provincial; y este último, al tiempo que se beneficiaba del prestigio que suponía asociarse con el CFI y el "DepEco" en el estímulo de la formación de posgrado, veía garantizada una vía para el reclutamiento de profesionales con formación especializada en el campo en el que le interesaba tallar, el de las finanzas públicas y el federalismo fiscal.

Los trabajos que conducirían a la creación de este posgrado se iniciaron a principios de la década de 1990, y el programa se inauguró en 1993, arrancando las promociones en 1994. En 1999, la Maestría fue categorizada por la Comisión Nacional de Evaluación y Acreditación Universitaria (CONEAU), recibiendo la máxima calificación (Departamento de Economía, Memoria 1992-2000).

Además del posgrado en el que comprometió financiamiento junto con el CFI, el Ministerio de Economía provincial acordó por convenio la asistencia de los investigadores del "DepEco" para el análisis de temas 
y cuestiones previamente definidos, colaboraciones que se produjeron a lo largo de la década de 1990, particularmente durante la gestión de Remes Lenicov. ${ }^{9}$

Pero esta colaboración no agotó las necesidades y demandas que el equipo económico provincial tenía en materia de análisis y producción de conocimiento. Si bien ella pudo encauzarse sobre la base de intereses relativamente compartidos en cuanto a temas, cuestiones y modos de abordaje, el "DepEco" en tanto institución académica tenía horizontes que no necesariamente coincidían con los del Ministerio de Economía provincial, y éste comenzó a distanciarse en términos de enfoques y prioridades hacia mediados de la década de 1990 y sobre todo desde que Remes Lenicov dejara el puesto de Ministro. ${ }^{10}$ La creación de una instancia propia de investigación y análisis, un espacio de trabajo adicional a los que ya existían dentro del Ministerio, fue el recurso al que el equipo económico provincial echó mano.

El Grupo de Investigación Económica (GIE) le dio forma y contenido al thinktank que el equipo económico provincial creó a su medida, a contrapelo de la tendencia generalizada a la externalización de la función de la capacidad analítica en centros y fundaciones que concentraron la palabra autorizada en materia de política económica y que, al decir de Heredia (2004), emergían como una suerte de "racionalidad paraestatal".

Si para los más viejos del equipo económico provincial el espejo en el que deseaban mirarse era el Instituto Di Tella de la década de 1960, la imagen dominante del thinktank que rondaba en la década de 1990 era la de la Fundación Mediterránea, y ésta más que otras usinas similares, porque había nutrido con su personal a las oficinas de la administración pública nacional. La experiencia del GIE dentro del Ministerio de Economía provincial se distanciaba de ellas no sólo por estar inscripta en las mismas estructuras del estado, sino por su direccionamiento político, del cual las otras renegaban aun cuando estuvieran preñadas ideológicamente. Además, se trataba de formar cuadros técnicos con visión de largo plazo que alimentarían la continuidad política del equipo económico provincial.

El GIE se integró con jóvenes economistas reclutados a partir de pruebas ad hoc. Bajo la supervisión de un coordinador en contacto directo con el Ministro, programaban su trabajo en temas de coyuntura y de carácter estructural que demandaban tiempos de investigación y análisis relativamente prolongados. Muchos de esos trabajos alimentaron las dos publicaciones impulsadas por el Ministerio de Economía provincial, las Noticias de Economía y los Cuadernos de Economía. Con fundamentos y métodos propios de la actividad académica, los integrantes del GIE aportaron al equipo económico provincial productos "a medida”, cuyos objetivos y contenidos eran controlados en su totalidad desde dentro del Ministerio y, por lo tanto, le brindaban márgenes de libertad significativos en relación con las voces expertas externas. Esta producción se validaba en el campo académico no sólo por la circulación de los integrantes del GIE en los grupos de investigación del "DepEco", sino por su participación en eventos científicos. ${ }^{11}$

Los integrantes del GIE fueron rotando según los diversos intereses repartidos entre oportunidades profesionales y académicas tanto dentro como fuera de la Provincia y del propio Ministerio de Economía provincial. Algunos de ellos se desarrollaron como funcionarios dentro de las oficinas del Ministerio, compartiendo la actividad con la docencia en las carreras de grado y posgrado de economía de la UNLP; otros se concentraron en la actividad académica; y otros, en fin, se radicaron en Estados Unidos a partir de los estudios de posgrado emprendidos en dicho país. ${ }^{12}$

Esta experiencia de incorporación y formación de jóvenes cuadros se encontró con algunos límites propios de las tensiones entre el desarrollo de las carreras sobre la base de intereses y perspectivas individuales, y los requerimientos del equipo y sus tramas jerárquicas. Es lo que está en la base del segundo de los factores que contribuyen a explicar el despliegue de la capacidad analítica en torno al Ministerio de Economía provincial, que denomino "brecha generacional". Por un lado, las primeras y segundas filas estaban ocupadas por la camada generacional que había dado los pasos iniciales en el proceso de formación del equipo económico provincial; por el otro, desde 1991 el Ministerio de Economía concentró su agenda en la cuestión de la hacienda y las finanzas públicas, abandonando el campo de la regulación y promoción de las actividades 
productivas. Como consecuencia de ello, algunos jóvenes economistas con perfiles ligados a la "economía real" y deseosos de impulsar sus carreras plantearon la apertura de otros horizontes.

El Centro de Estudios Bonaerenses (CEB) fue creado en 1991, ${ }^{13}$ y agrupó a economistas formados en la UNLP y en el trabajo dentro del Ministerio de Economía provincial que se concentraron en la realización de análisis de carácter estructural y coyuntural sobre la economía nacional, con particular referencia a la situación de la Provincia de Buenos Aires. Su actividad incluyó la publicación de informes mensuales que, además de una nota editorial, contenían uno o dos trabajos de análisis sectorial de la economía, y secciones fijas con indicadores de actividad económica nacional y provincial, en consonancia con las pautas por entonces dominantes para este tipo de piezas. El financiamiento se obtenía por medio de suscripciones, pero también por contratos del CFI gestionados desde el Ministerio de Economía provincial, lo que pone de relieve la relevancia del vínculo entre la novel empresa y la vieja institución estatal en cuyo seno fue imaginada.

El CEB buscó proyectarse en el nivel nacional que constituía el horizonte de sus trabajos. Pero esa proyección tuvo como seña de identidad a la Provincia de Buenos Aires, el punto de referencia desde donde se producía el trabajo de análisis. ${ }^{14}$

Además de la actividad de investigación y análisis, el CEB promovió, teniendo como modelo al Instituto de Desarrollo Económico y Social (IDES), ${ }^{15}$ la actividad de formación por medio de la realización de seminarios internacionales con profesores del Instituto Latinoamericano y del Caribe de Planificación Económica y Social (ILPES) -dependiente de la Comisión Económica para América Latina y el Caribe (CEPAL) y con sede en Chile-, y cursos a cargo de reconocidos cientistas sociales argentinos y sobre todo economistas, como Susana Torrado, Luis Beccaria, Francisco Gato, Roberto Frenkel, Guillermo Rozenwurcel, todos ellos referentes críticos de las políticas neoliberales desplegadas durante la década de 1990.

La experiencia del Instituto de Estudios Fiscales y Económicos (IEFE) habla del tercero de los factores que explica el desplieguede la capacidad analítica alrededor del -y en relación con- Ministerio de Economía provincial: la "brecha ideológica". Con ello hago referencia a una de las fisuras que experimentó tempranamente el equipo económico provincial, como consecuencia del viraje del peronismo en la década de 1990 y los reacomodamientos que ello supuso entre los economistas platenses que habían protagonizado la experiencia de la renovación peronista desde mediados de la década anterior. En ese marco afloraron perspectivas distintas en torno al ritmo e intensidad de las intervenciones -y también su dirección- al calor de la hiperinflación de 1989, el rumbo adoptado por el presidente Carlos Menem y las adaptaciones que experimentó el justicialismo provincial conducido por Eduardo Duhalde, gobernador desde 1991. Los críticos del nuevo rumbo buscaron un espacio propio que permitiera canalizar sus posiciones y construir una identidad que, sin suponer una ruptura total con el colectivo mayor que los contenía, preservara las notas distintivas de una concepción transformadora. El IEFE constituyó el vehículo para ello.

Los descontentos que ya no encontraban lugar dentro del Ministerio de Economía Provincial -como José Sbatella y Ricardo Angelucci-, pero también quienes seguían siendo parte del equipo económico provincial - como Sergio Bugallo, Mónica Carné, Elsa Pérez y Carlos Lapine- integraron el staff de la "Mediterránea buena", tal como caracterizó al IEFE uno de sus integrantes.

A semejanza del CEB, el IEFE combinó el trabajo analítico con el de consultoría, abordando los temas y problemas a escala nacional y provincial, y poniendo el énfasis en las finanzas públicas. Se financiaba por medio de suscripciones y contratos para el desarrollo de asistencia técnica en el campo tributario y de las finanzas públicas, particularmente en el nivel subnacional. Publicaba un informe mensual que contaba con una editorial en la que se realizaba una caracterización de la escena política nacional, y secciones fijas que abordaban temas relevantes de la economía y las finanzas públicas en general y de la Provincia de Buenos Aires en particular, y el seguimiento de indicadores seleccionados sobre el comportamiento de las variables económicas, fiscales y tributarias.

El colectivo tuvo una fortaleza en el análisis fiscal porque muchos de sus integrantes trabajaban en paralelo en el Ministerio de Economía provincial, y contaban con fuentes cuyo acceso no era sencillo. Esto permite 
aventurar que el IEFE constituía una suerte de alter ego del Ministerio de Economía, un espacio en el que era posible decir cosas y realizar críticas que estaban vedadas desde la posición institucional de gobierno. Buena información combinada con mayores grados de libertad para su tratamiento e interpretación, componían una fórmula que brindó al colectivo del IEFE un lugar en el mundo del análisis económico local, y le proveyó la base a partir de la cual construyó su trayectoria en ese campo. ${ }^{16}$ Esa fortaleza se vio impulsada a partir de la incorporación, junto con Jorge Carrera, un economista graduado en la UNLP y doctorado en Italia, de una camada de jóvenes economistas que le imprimieron a la revista un perfil más académico y contribuyeron a consolidar la producción intelectual del colectivo. ${ }^{17}$

Adentro y afuera del Ministerio de Economía provincial, los economistas platenses desplegaron capacidad analítica. Lo hicieron para apoyar las posiciones y políticas que el Ministerio impulsaba y sostenía, pero también para discutirlas y discutir la política económica nacional. De una u otra forma, todos compartían la convicción de que el conocimiento ocupaba un lugar central en el desarrollo de las políticas públicas, brindándoles sus fundamentos y argumentos. Todos ellos reconocían los problemas en la producción de conocimiento, y buscaron por estas diversas vías resolverlos. Todos ellos, en fin, deseaban intervenir en los debates sobre los principales aspectos de la economía y la política económica nacional y provincial, teniendo como referentes a usinas de pensamiento que eran tan reconocidas como cuestionadas. La producción de estos espacios -el GIE dentro del Ministerio, el CEB y el IEFE fuera de él- tenía, no obstante el carácter "universal" de las categorías y conceptos empleados para su desarrollo, una marca bonaerense, platense.

en la provincia de Buenos Aires hubo una intención, una pelea, una disputa, por tratar de generar un pensamiento crítico. Independientemente de estas divisiones o estas diferencias ... la provincia de Buenos Aires, o el grupo por lo menos ... en el que a uno le tocó estar más adentro o más afuera, siempre tuvo una bandera, que ... fue que no alcanza con tener gerentes en la administración pública; hacen falta ... tipos que tengan la capacidad de discutir, de analizar distintos caminos, y tomar un camino, que para alguno habrá sido equivocado, para otro habrá sido el acertado ... pero siempre a partir de un análisis crítico. O sea, ninguno de todos los que [protagonizaron la experiencia bajo análisis] ... son tipos que han pasado simplemente para agarrar un papel, firmarlo, y hacer el tránsito normal de la burocracia (ex funcionario del Ministerio de Economía, miembro de IEFE).

Este es el contexto en el que se inscribió la producción específica impulsada por el equipo económico provincial dentro del Ministerio de Economía: las Noticias de Economia y los Cuadernos de Economía. A su análisis está dedicado el próximo apartado.

\section{LA PRODUCCIÓN DE CONOCIMIENTO PARA LAS POLÍTICAS: LAS Noticias de ECONOMÍA Y LOS Cuadernos de Economía}

Las investigaciones del GIE y los equipos del Departamento de Economía de la Facultad de Ciencias Económicas de la UNLP contribuyeron a alimentar dos publicaciones que en forma prácticamente simultánea el equipo económico provincial impulsó y sostuvo \#aunque con altibajos- a lo largo de los 20 años de su actuación: las Noticias de Economía y los Cuadernos de Economía.

En ambas es posible distinguir una primera época que se corresponde con los 8 años de gestión del Ministerio de Economía a cargo de Jorge Remes Lenicov -de mediados de 1989 a mediados de 1997-, durante la cual se consolidaron y tuvieron una notable continuidad. La segunda época, correspondiente a las gestiones de Jorge Sarghini y Gerardo Otero, abarca el resto del período considerado, caracterizado por la diversificación de temas, problemas y formatos -esto en el caso de las Noticias de Economía- así como por las dificultades para sostener la periodicidad inicialmente planteada en ambas publicaciones (mensual para los Cuadernos y bimestral para las Noticias).

La publicación de las Noticias de Economía se inició a mediados de 1989 con el propósito de difundir la actividad del Ministerio, las principales medidas de política adoptadas tanto en su ámbito como en la escala provincial general e incluso en la nacional, y las posiciones relacionadas con temas y cuestiones directa e 
indirectamente relacionados con su quehacer. La revista alcanzaba por lo general entre las 20 y las 40 páginas, y una tirada de entre 1.000 y 1.500 ejemplares. Estaba dirigida fundamentalmente a las autoridades provinciales de los tres poderes y a las municipales, pero también era recibida por las diversas entidades representativas de la actividad económica provincial, y las asociaciones y colegios profesionales. ${ }^{18}$

La revista tuvo una estructura bastante homogénea, aun cuando fueron introduciéndose modificaciones e innovaciones en el tipo de información producida o a difundir, particularmente en los números publicados durante la gestión de Otero. Cada número era abierto con una editorial firmada por el ministro, en la que se hacía referencia a los temas o cuestiones relevantes desde la perspectiva del Ministerio; ${ }^{19}$ luego la información era organizada en secciones más o menos fijas que se consolidaron a partir del № 15 (enefeb 1992): "equidad contributiva y control de la evasión” concentraba las noticias referidas a la política y administración tributaria, "transformación del estado", las correspondientes a los avances en relación con la reforma del estado provincial y la evolución de su presupuesto; y "federalismo fiscal", las vinculadas con el reparto de los recursos tanto entre Nación y provincias, como entre la Provincia y los municipios. ${ }^{20} \mathrm{~A}$ partir del No26 (nov-dic 1993) se incorporó una sección denominada “economía y sociedad”, que a veces reemplazaba a la de "federalismo fiscal".

En su segunda época, la revista mantuvo la estructura y desde el $\mathrm{N}^{\circ} 66$ (jun-jul de 2003) continuó con secciones que, sin portar un nombre, daban cuenta de contenidos similares a los de la primera época, incorporándose de manera más evidente los relacionados con la actividad económica provincial. Además apareció una sección denominada "Fichero" en la que se difundían indicadores y apostillas de intervenciones o reflexiones de dirigentes nacionales, latinoamericanos e internacionales, y notas y reseñas sobre exponentes del pensamiento económico, poniendo de manifiesto el cambio de época relacionado con el mejoramiento del desempeño general tanto del país como de América Latina, así como con el renovado escenario político latinoamericano y el reflujo del pensamiento neoliberal. ${ }^{21}$

En esta segunda época las páginas de la revista sirvieron también para difundir la producción de la Dirección Provincial de Estadísticas, que desde 2001 había vuelto a la órbita del Ministerio de Economía, revitalizando el trabajo estadístico provincial. Así, en las Noticias 68 a 70 se incluyeron artículos sobre el comportamiento demográfico provincial, y desde la Noticias 69 se incorporó como sección "Indicadores de la actividad económica bonaerense", elaborada por el GIE.

Otro rasgo destacable de esta publicación reside en la inclusión de numerosas intervenciones de los funcionarios del Ministerio -y particularmente del Ministro- en foros, seminarios, jornadas y debates, de artículos publicados en la prensa o bien específicamente elaborados para la revista con el apoyo del GIE. Si en la primera época los temas que ocuparon la escena central de este tipo de intervenciones públicas fueron la cuestión de la coparticipación federal de impuestos, y la de la transformación del estado, desde 1997 en adelante los mismos fueron acompañados por consideraciones y reflexiones sobre la "economía real” y la escena internacional.

La revista tenía un claro sesgo institucional, y desde ese lugar la presentación de la información guardaba equilibrios en función del público al que estaba destinada: la preocupación permanente de los miembros del equipo económico provincial por la racionalidad del gasto público, su pertinencia y adecuada orientación, en algunas ocasiones se topaba con la lógica política cercana al juego del "barril sin fondo" ${ }^{22}$

Las Noticias de Economía constituyeron, en fin, una tribuna desde la cual el equipo económico provincial construyó su presencia y autoridad en el ámbito provincial y nacional, buscando comunicar el sentido de sus medidas y la perspectiva política que las orientaban, así como influir, desde un lenguaje relativamente sencillo y con intención pedagógica, en el reconocimiento y toma de conciencia por parte de la plana política en torno a los que eran considerados los ejes centrales de la intervención pública provincial.

Por su parte, la creación de los Cuadernos de Economia en 1994 pone de relieve el interés por difundir los estudios y trabajos cuyo carácter y profundidad excedían la posibilidad de su publicación en las Noticias 
de Economía. Los Cuadernos fueron concebidos como un espacio para discutir orientaciones y tendencias vinculadas con los asuntos públicos no sólo de carácter económico y provincial, sino de una mayor diversidad de áreas de intervención, y con alcance nacional. Los estudios podían ser producto del trabajo interno del Ministerio, de los acuerdos con la Facultad de Ciencias Económicas de la UNLP, o de la actividad financiada con aportes del Programa de Naciones Unidas para el Desarrollo (PNUD). Los contenidos y orientaciones de los estudios publicados, que no expresaban necesariamente las posiciones del Ministerio, contribuirían a promover la discusión sobre "las futuras políticas a implementar" (Cuadernos 1: 4). ${ }^{23}$

A diferencia de otras publicaciones periódicas, los Cuadernos se caracterizaron por el abordaje monográfico de los temas propuestos en cada número, desde un estilo que, en la mayor parte de los casos, guarda las convenciones científicas y académicas de presentación de resultados de investigación.

El análisis de los temas contemplados permite apreciar la preponderancia otorgada a aspectos teóricos y aplicados del funcionamiento de la economía tanto nacional como provincial, y las alternativas de política económica. Dentro de ello, las finanzas públicas como recorte específico compartían su lugar con números dedicados al análisis del sector industrial (nacional y provincial), la actividad exportadora, la dinámica del empleo, el mercado de trabajo y la distribución del ingreso, el comportamiento del ciclo económico, los problemas del desarrollo regional, el problema de la regulación de servicios públicos. Asimismo, las políticas sociales - particularmente salud y educación- recibieron un espacio relativamente importante, así como cuestiones vinculados con la reforma y organización del estado y, en menor medida, la problemática ambiental.

Siguiendo con la distinción practicada en el análisis de las Noticias de Economía, en la primera época, correspondiente a la gestión de Remes Lenicov, la preponderancia de los números dedicados a aspectos de las finanzas públicas y el federalismo fiscal fue de la mano con la intensa colaboración de los equipos de investigación del "DepEco", en el marco del convenio suscripto entre el mismo y el Ministerio de Economía provincial. En la segunda época, en cambio, la diversidad temática fue mayor, y al igual que en el caso de las Noticias de Economia, ganaron preponderancia los temas y problemas vinculados con la "economía real", en estrecha asociación con el relativo declive de la participación de los investigadores de la UNLP y el creciente predominio de los equipos propios nucleados en el GIE.

Por el carácter académico de esta publicación y el modo en que eran tratados los diversos temas abordados, el público al que estaba destinada debía poseer un piso de conocimiento de la economía relativamente especializado. El desarrollo de gran parte de los temas hacía gala de los conceptos y métodos de la disciplina en sus vertientes modelizantes y matematizantes, poniendo límites de acceso a los profanos. Pero a la vez, los Cuadernos constituían una herramienta de trabajo para los miembros del equipo económico provincial, en varios sentidos: eran fuente no sólo de información sino también de prestigio. Por lo demás, los Cuadernos tuvieron una circulación similar a la delas revistas académicas en términos de alcances y límites, y su valoración era alta en los medios profesionales y académicos. ${ }^{24}$

Como señalé anteriormente, los referentes académicos del "DepEco" tuvieron una mayor presencia relativa en la primera época, y su participación disminuyó a medida que se avanza en el tiempo, tendiendo a predominar los estudios realizados desde el mismo Ministerio. Ello da cuenta de una suerte de división del trabajo y su distribución en el tiempo relacionada tanto con la especialización relativa de ambos grupos, como con los intereses y preocupaciones que el Ministerio de Economía fue desplegando en relación con el desarrollo económico entendido en sentido amplio y, me atrevería a decir, estructural: los equipos del "DepEco" mostraban solidez en el campo de las finanzas públicas y la economía del sector público en general, pero los problemas y cuestiones de la producción de bienes y servicios parecían caer por fuera de su especialización. Y es en estos problemas y cuestiones en los que los equipos del propio Ministerio de Economía, nucleados en el GIE, tendieron a tallar.

La cuestión fiscal y tributaria ocupa un lugar central en la primera etapa de la colección, alternando con otros temas vinculados con el desarrollo económico provincial y nacional, que van adquiriendo preeminencia 
progresiva. ${ }^{25}$ Esto no quiere decir, sin embargo, que dicha cuestión no estuviera contemplada en la segunda época, ni que los equipos del Ministerio de Economía provincial no intervinieran con aportes. ${ }^{26}$

Desde el No 42 en adelante, cuando los trabajos son producidos por miembros del GIE, los diversos ministros aparecen coordinándolos, y el grueso de los mismos está dedicado al análisis de la economía provincial y dimensiones relevantes de la organización y funcionamiento de la economía. ${ }^{27} \mathrm{Al}$ igual que en el caso de la cuestión fiscal y tributaria, esto no implica que los equipos del "DepEco" no abordaran aspectos de la "economía real”, ni que los mismos estuvieran ausentes en la primera época. ${ }^{28}$

Pero si estos puentes temáticos hablan de la versatilidad existente en los economistas de uno y otro lado y contribuyen a atenuar la especialización relativa identificada, no suponen uniformidad en los enfoques adoptados: en el caso de los investigadores del "DepEco", los análisis descansan en los supuestos de la matriz neoclásica y sus diversas variantes relacionadas con las teorías de los costos de transacción y de la elección racional, y la teoría del federalismo fiscal en su formulación clásica, que prácticamente no enfrenta teorías rivales. Los trabajos de los equipos internos del Ministerio, por su parte, abrevan en perspectivas que, sin abandonar en todos los casos los aportes de la teoría neoclásica y en particular del federalismo fiscal, se inscriben en aproximaciones críticas y "heterodoxas" respecto de la "corriente principal". 29

Los Cuadernos no dejaron aspecto de la realidad social sin abordar, expresando las preocupaciones e intereses que el Ministerio de Economía buscó promover e instalar en el debate público. Ellos ponen de relieve la vocación del saber económico por abarcar tan diversos aspectos de esa realidad social desde la sola y particular perspectiva que se deriva de sus premisas y supuestos, aun cuando éstos fueran objeto de disputa dentro del propio campo disciplinario. En este sentido, los Cuadernos no se apartaron de la impronta general que caracterizó al saber experto en economía desde la segunda mitad del siglo XX en adelante, y particularmente desde la crisis de 1970: su poderoso influjo y capacidad para moldear la comprensión y visión del mundo desde un lenguaje y una perspectiva que demanda conocimientos específicos no intercambiables con otros, y no disponibles para profanos.

Las Noticias y los Cuadernos de Economía condensaron, en conjunto, la producción de conocimiento que el Ministerio de Economía provincial contribuyó a desarrollar por medio de sus propios recursos -la formación de grupos internos de investigación y análisis como el GIE y los consultores contratados bajo programas específicos- o estimulando la disposición de recursos externos - como los del Departamento de Economía de la Facultad de Ciencias Económicas de la UNLP-. Con sesgos definidos -informativo y formativo en el primer caso, académico y respetuoso de las reglas del trabajo científico en el segundo-, las publicaciones buscaron colocar cuestiones en la agenda y someter ideas al debate tanto dentro del propio campo disciplinar como del campo más general de las políticas públicas. Al mismo tiempo, constituyeron la "carta de presentación" del equipo económico provincial.

\section{Reflexiones finales}

La alta valoración del conocimiento como soporte de las intervenciones públicas fue compartida por todos los economistas que de manera directa e indirecta tuvieron relación con el Ministerio de Economía provincial, de suerte que puede afirmarse que la producción de capacidad analítica fue algo que lo tuvo como epicentro, aunque su demanda e impulso no agotó el despliegue que los economistas platenses protagonizaron en este campo. Adentro y afuera del Ministerio de Economía, y en asociación con el principal centro académico que ofició como semillero, los economistas platenses produjeron conocimiento en el convencimiento de que ello constituía una llave decisiva para el desarrollo de las políticas. Junto con el equipo económico provincial, los equipos del "DepEco", los jóvenes economistas que integraron el GIE, y los que dieron vida al CEB y al IEFE, todos ellos contribuyeron al desarrollo de la intelligentsia técnica que sería un sello distintivo de la escena 
política bonaerense. Un "pensamiento crítico", al decir de uno de los protagonistas de esta experiencia, que buscó tallar en el debate de las ideas sobre economía y política económica.

El conocimiento que estuvo en la base de la producción de capacidad analítica, aun cuando abrevara en la experiencia de gestión o dialogara con ella, respetó los estándares propios del quehacer científico-técnico. Las mediaciones entre las esferas del mundo científico-técnico y la de política pública podían tener zonas grises así como un carácter no lineal ni inmediato, y en ellas el equipo económico provincial se posicionaba como un traductor dotado de destrezas específicas.

Conocimiento y racionalidad eran los organizadores por excelencia de la perspectiva del equipo económico provincial, y eran movilizados en sus relaciones e intervenciones dentro del gobierno provincial y con el gobierno nacional. Los instrumentos de comunicación construidos, las Noticias y los Cuadernos de Economía, albergaban de manera armónica esta combinación: si en las primeras la cuidadosa y sistemática exposición de las actuaciones colocaba en el centro de la escena a la racionalidad como herramienta política, los segundos fueron el espacio privilegiado de construcción del conocimiento requerido para dotar a esa racionalidad (política) de sustancia y fundamentos.

La experiencia del equipo económico en la gestión provincial no sólo se nutrió de estos supuestos, sino también de la militancia política en el seno del justicialismo provincial, lo que permite delinear los contornos y contenidos de la relación entre conocimiento y política, sus dinámicas y mediaciones. El perfil político resultante se distancia en parte del que típicamente se desprende de trabajo u oficio político tal como es caracterizado por Michel Offerlé(2011), pero también del que ha sido propuesto bajo la figura del "tecnopolítico" (Domínguez, 1997), toda vez que la militancia política y el carácter colectivo de la actuación encuadrada dentro de un partido político le imprime rasgos diferenciales.

Los economistas devenidos actores estratégicos con importantes recursos de poder, consagraron una perspectiva que revistió la condición de ortodoxia y unos espacios "para-estatales" -los thinktanks- en los que se concentró la capacidad analítica para la orientación de las políticas públicas. En una suerte de contrapunto, la experiencia bonaerense transitó por carriles que pusieron de manifiesto una concepción distinta, aunque no exenta de tensiones y ambigüedades, en el desarrollo de ese papel estratégico.Los grupos investigación dentro del Ministerio de Economía provincial, y los equipos congregados en el "DepEco", en el CEB, en el IEFE, expresaron la vitalidad de la escena bonaerense -más bien platense- para generar un pensamiento propio, de carácter crítico, en un contexto dominado por la perspectiva neoclásica y en el campo de las políticas económicas, las reformas estructurales asociadas con el Consenso de Washington.

"Mediterráneas buenas", y espacios estatales de producción de conocimiento: tal fue la impronta que caracterizó a estas empresas y que ponía de relieve al menos tres cosas. En primer lugar, la pretensión de tomar distancia respecto del "pensamiento único", poniendo en cuestión su pretendido carácter apolítico al tiempo que reivindicando la reunión de análisis fundado y perspectiva política. En segundo lugar, la preocupación por la formación de cuadros técnicos dentro del estado, lo que involucraba que el conocimiento especializado se revestía de ese sesgo, y en consecuencia adquiría contornos precisos y no intercambiables con los de otras usinas de pensamiento. Y por último, que el equipo económico provincial se hallaba inscripto en una red de producción de conocimiento que le amplificaba el rango de intervención y reflexión sobre cuestiones económicas provinciales y nacionales, toda vez que los thinktanks locales -las "mediterráneas buenas"- fungían como una suerte de alter ego, expresando en muchas ocasiones posiciones y críticas a la escena política y económica nacional y provincial que por la responsabilidad institucional los miembros del equipo económico provincial estaban inhibidos de explicitar.

En el entrelazamiento de estas cosas es posible advertir las tensiones y ambigüedades que caracterizaron a estas empresas: se miraban en el espejo de thinktanks que admiraban y al mismo tiempo objetaban; buscaban producir un pensamiento bonaerense con una impronta propia, distante y crítica del "pensamiento único" de cuño neoclásico, pero al mismo tiempo eran tributarias de muchas de sus premisas; se inscribían, en fin, 
en unas relaciones de pertenencia cruzada con el "DepEco" y el Ministerio de Economía provincial, dentro de las cuales circulaban recursos, lealtades, compromisos, valores y creencias, y sentido político.

En ese contexto el equipo económico provincial -y especialmente uno de sus principales referentes, Remes Lenicov- impulsó las Noticias y los Cuadernos de Economía, dos publicaciones con diverso contenido y alcance que constituyeron su "carta de presentación". La "conciencia" sobre la Provincia de Buenos Aires y las finanzas provinciales anidaba allí, condensada en producciones que pendulaban entre la información y la formación, entre la tribuna política y la academia, y que expresaban, en fin, el activo que el equipo económico provincial tenía para ofrecer en la escena política provincial, aquello que lo diferenciaba de cualquier otro actor político en esa escena.

\section{BiBLIOGRAFÍA Y FUENTES ${ }^{30}$}

Aguilar Villanueva, Luis (1994).La Implementación de Politicas Públicas. México: Porrúa.

Biglaiser, Glen (2002). "The Internationalization of Chicago's Economics in Latin America”. The University of Chicago.

Camou, Antonio (1997). "Los consejeros del príncipe. Saber técnico y política en los procesos de reforma económica en América Latina”. Nueva Sociedad 152.

Camou, Antonio (1998). "Saber técnico y política en los orígenes del menemismo". Perfiles Latinoamericanos 12.

Camou, Antonio (2006). "El Saber detrás del Trono. Intelectuales-expertos, tanques de pensamiento y políticas económicas en la Argentina democrática (1985-2001)”. En Garcé, Adolfo y GerardoUña (comps.) Thinktanks y políticas públicas en Latinoamérica. Buenos Aires: Prometeo.

Conaghan, C., J. Malloy y L. Abugattas (1990)."Business and the Boys: The politics of Neoliberalism in the Central Andes" LARR 25 (2).

Chudnovsky, Mariana (2014) "Un modelo analítico para estudiar las capacidades estatales subnacionales. El rol de los gabinetes en las políticas sanitarias de las provincias argentinas de Chaco y Formosa”. En Walter, Jorge y Diego Pando (comps.) Planificación estratégica. Nuevos desafios y enfoques en el ámbito público. Buenos Aires: Sociedad Argentina de Análisis Político - Universidad de San Andrés.

Departamento de Economía, Memorias 1992-2000, 2001, 2002, 2003, 2004, 2005, 2006 y 2007. Universidad Nacional de La Plata, Facultad de Ciencias Económicas.

Domínguez, Jorge (ed.) (1997).Technopols: Freeing Politics and Markets in Latin Americain the 1990s. University Park, PA: The Pennsylvania State University Press.

Entrevistas semi-estructuradas realizadas entre 2012 y 2014 a integrantes del equipo económico provincial y referentes del campo político y académico provincial.

Fourcade-Gourinchas, Marion y Sarah Babb (2002). "The Rebirth of the Liberal Creed: Paths to Neoliberalism in Four Countries". The American Journal of Sociology 108 (3).

Heredia, Mariana (2004). "El Proceso como bisagra. Emergencia y consolidación del liberalismo tecnocrático en la Argentina”. En Pucciarelli, Alfredo (comp.) Empresarios, tecnócratasy militares. La trama corporativa de la última dictadura militar. Buenos Aires: Siglo XXI.

Heredia, Mariana (2006). "La demarcación de la frontera entre economía y política en democracia. Actores y controversias en torno de la política económica de Alfonsín”. En Pucciarelli, Alfredo (coord.).Los años de Alfonsin ¿Elpoder de la democracia o la democracia del poder? Buenos Aires: Siglo XXI.

Heredia, Mariana (2008). "Entre reflexividad, legitimación y performatividad. El discurso económico en la instauración y la crisis de la convertibilidad". Critica en Desarrollo 2.

Heredia, Mariana (2015).Cuando los economistas alcanzaron el poder. Buenos Aires: Siglo XXI.

Loureiro, María Rita (2006). “A participação dos economistas no governo”. Análise 17 (2). Porto Alegre.

Ministerio de Economía de la Provincia de Buenos Aires: Cuadernos de Economía Nros. 1 a 75.

Ministerio de Economía de la Provincia de Buenos Aires: Noticias de Economía Nros. 1 a 77. 
Montecinos, Verónica (1997). "El valor simbólico de los economistas en la democratización de la política chilena". Nueva Sociedad 152.

Morresi, Sergio y Gabriel Vommaro (comps.) (2011).Saber lo que se hace. Expertos y política en Argentina. Buenos Aires: Prometeo.

Offerlé, Michel (2011). "Los oficios, la profesión y la vocación de la política”. PolHis 7.

Pereyra, Elsa (2008) "Política y burocracia en procesos de reforma de los estados provinciales en la década de 1990. Un análisis desde los Programas Provincias I y II". Tesis de Maestría. Programa de Administración y Políticas Públicas. Universidad de San Andrés.

Pereyra, Elsa (2016). "Elencos en el nivel subnacional: trayectorias y saberes en la experiencia de losintegrantes del Ministerio de Economía de la Provincia de Buenos Aires (1987-2007)”. VI Jornadas de Estudios Políticos Los usos del Estado. Proyectos políticos en disputa en las democracias de América Latina. 25 y 26 de agosto de 2016. Los Polvorines: Universidad Nacional de General Sarmiento.

Pereyra, Elsa (2017).“Trayectorias y saberes en la provincia de Buenos Aires: El equipo del Ministerio de Economía (1987-2007)". Estudios Sociales del Estado 3 (6).

Ramírez, Hernán (2001). "La Fundación Mediterránea y de cómo construir poder: La irrupción del Interior en el diseño de la política económica argentina”. História Econômica \& História de Empresas 4 (2): 131-62.

Ramírez, Hernán (2007). Corporaciones en el poder. Institutos económicos y acción política en Brasil y Argentina: IPÊS, FIEL y Fundación Mediterránea. Buenos Aires: Lenguaje Claro Editora.

Santiso, Javier y Lawrence Whitehead (2006)."Ulysses, the Sirens and the Art of Navigation: Political and Technical Rationality in Latin America”. Paris: OECD, WorkingPaperNo 256.

Serafinoff, Valeria (2014) "Patrones de relación intergubernamental en la formulacióne implementación de políticas públicas en Argentina”. Mimeo.

\section{Notas}

1 Este trabajo se apoya en mi tesis de doctorado: "Burocracias especializadas en el nivel subnacional: El equipo del Ministerio de Economía de la Provincia de Buenos Aires (1987-2007)”. Doctorado de la Facultad de Ciencias Sociales de la Universidad de Buenos Aires (2017). Una primera versión fue presentada en las XVI Jornadas Interescuelas y Departamentos de Historia, desarrolladas en Mar del Plata entre el 9 y el 11 de agosto de 2017. Agradezco los comentarios de Andrés Regalsky y Aníbal Jáuregui realizados en esa oportunidad, así como los del/la evaluador/a anónimo/a.

2 Por cierto no fue únicamente en el campo de la economía que el saber especializado cumplió con esta funciónestratégica. Antes bien, todas las intervenciones públicas del estado lo demandaron.

3 La influencia de las universidades norteamericanas en la modelación de las perspectivas dominantes se halla ampliamente documentada, pudiendo consultarse los siguientes trabajos: Conaghan, Malloy y Abugattas, 1990; Domínguez, 1997; Montecinos, 1997; Biglaiser, 2002; Fourcade-Gourinchas y Babb, 2002; Heredia, 2004; Loureiro, 2006; Camou, 2006; Santiso y Whitehead, 2006.

4 Para ello, ver entre otros, los trabajos de Mariana Heredia (2006, 2008 y 2015) sobre los economistas, los de Antonio Camou (1997, 1998 y 2006) sobre el saber técnico y los thinktanks, y los de Hernán Ramírez (2001 y 2007) sobre la Fundación Mediterránea y FIEL. En cuanto a los estados provinciales, los modos de construcción y ejercicio del poder político y el papel que el saber especializado juega en ello, es posible afirmar que nuestro conocimiento sobre tales dinámicas y procesos es significativamente escaso, si no inexistente. En mi investigación pude identificar algunas líneas de trabajo relevantes: el análisis político-institucional, que discute la formación y comportamiento de los aparatos burocrático-administrativos; los estudios sobre federalismo fiscal, con énfasis en la coparticipación federal de impuestos; y los estudios sobre el régimen federal de gobierno y los sistemas políticos provinciales, que procuran indagar en torno de los problemas de la construcción de regímenes democráticos en dichos espacios subnacionales. En todas ellas predomina el par conceptual "nación-provincias", a partir del cual el primer término, la Nación, suele aparecer enfrentado con el segundo, las provincias, o bien como modelo implícito para ponderar eventuales déficits de carácter político-institucional. Asimismo, la relación entre técnica y política está prácticamente inexplorada. Ver, para mayores detalles, Pereyra (2008 y 2017), Serafinoff(2014) y Chudnovski (2014). 
5 Si esta calificación es quizás excesiva para la experiencia del Di Tella, que tuvo un sesgo más bien académico, fue sin dudas la predominante para referir a los denominados thinktanks que por esa misma época surgieron en nuestro país, comola Fundación Mediterránea, la Fundación de Investigaciones Económicas Latinoamericanas (FIEL) y el Centro de Estudios Macroeconómicos de la Argentina (CEMA).

6 Estas direcciones provinciales estuvieron a cargo de economistas que luego ocuparon posiciones de relevancia: la Dirección Provincial de Estudios Económicos fue la actividad a cargo de Remes Lenicov cuando ingresó al Ministerio de Economía durante la gestión radical (entre 1983 y 1986), y más adelante, condujeron la oficina Alejandro Arlía (1997-2001) y Silvina Batakis (2001-2002). Por su parte, la Dirección Provincial de Estudios Fiscales fue dirigida por Jorge Sarghini en los dos primeros años del gobierno de Antonio Cafiero (1987-1989). Estos cuatro economistas ocuparon el máximo cargo del Ministerio de Economía, dos de ellos dentro de los 20 años considerados por mi investigación (Remes Lenicov 1989-1997 y Sarghini 1997-2002), y los otros dos durante el gobierno de Daniel Scioli (Arlía 2009-2011 y Batakis 2011-2015).

7 Ambas vías podían confluir, y de hecho muchos graduados que transitaron el programa de posgrado luego recalaron en el Ministerio.

8 Horacio Pereyra, profesor titular de "Introducción a las Ciencias Sociales" hacia fines de la década de 1960 y principios de la de 1970, materia común a las carreras de la Facultad de Ciencias Económicas de la UNLP, fue su decano en el tumultuoso año 1973, y su gestión duró muy poco como consecuencia de enfrentamientos con los representantes más radicalizados de las agrupaciones peronistas de izquierda. Fue declarado prescindible durante la dictadura de 1976-83 y con el regreso de la democracia, recuperó sus cargos tanto en la UNLP como en la UBA. Alberto Porto es un economista de dilatada trayectoria académica, referencia en los estudios sobre federalismo fiscal, cuya carrera se desarrolló casi exclusivamente en la UNLP desde que egresó y se doctoró como economista en esa misma casa de estudios, y en menor medida en la UTDT. Jorge Remes Lenicov fue Jefe del Departamento de Economía y Secretario Académico de la Facultad de Ciencias Económicas en 1973. Exiliado en el primer tramo de la dictadura militar de 1976-83, a su regreso se incorporó a la Fundación de Investigaciones para el Desarrollo (FIDE), y desde la recuperación de la democracia, retomó la actividad académica en la UNLP y la actividad profesional en el Ministerio de Economía provincial. Tanto antes como después de la dictadura militar del 76-83 -que constituyó una bisagra-, los vínculos entre Pereyra, Porto y Remes Lenicov fueron suficientemente sostenidos -sobre todo entre Pereyra y Remes Lenicov, y entre éste y Portocomo para desembocar en el proyecto del posgrado.

9 Como fruto de ello, los investigadores realizaron 100 informes.El detalle aparece en la página del Departamento de Economía, Centro de Estudios en Finanzas Publicas (CEFIP), ex Instituto de Investigaciones de la Maestría en Finanzas Públicas Provinciales y Municipales: http://www.depeco.econo.unlp.edu.ar/cefip-estudios-fiscales.php [visita 25-02-2016]. Algunos de ellos serían publicados como Cuadernos de Economía (ver la próxima sección). Curiosamente no se consigna el período de la colaboración en el marco de los convenios que se renovaron periódicamente, aunque puede inferirse por algunas fechas que figuran en los primeros y últimos informes, que ella se verificó entre 1990/91 y 2001/2002.

10 Realizo un desarrollo más pormenorizado de estas cuestiones en el Capítulo 5 de mi tesis.

11 “a nosotros nos preocupó ... tener una evaluación externa de lo que hacíamos, entonces nos presentábamos a concursos ... generalmente en todos los años hay algún trabajo nuestro o en la Asociación de Economía Política, o en las Jornadas de Finanzas Públicas de Córdoba...Y nos presentamos después al concurso del Consejo Profesional de Ciencias Económicas de Capital, que era un concurso muy conocido en ese momento, y llevamos uno de los trabajos, que es hoy un Cuaderno de Economía, y ganamos el primer premio" (funcionario del Ministerio de Economía, tercera línea política tributaria).Se trató del concurso de 1999 organizado por el referido Consejo Profesional de Ciencias Económicas, en que el equipo integrado por Ariel Aramburu, Agustín Lódola, Fernando Cuenin, Federico Cerimedo, Matías Busso y Diego Moccero, obtuvo el Primer Premio "Manuel Belgrano" por el trabajo “Crecimiento sostenido y con equidad. El rol del financiamiento" (Departamento de Economía, Memoria 1992-2000).

12 Un listado no exhaustivo incluye los siguientes economistas: quienes desarrollaron carrera en el Ministerio de Economía: Agustín Lódola, Federico Cerimedo, Pedro Aramburu, León Salim; quienes se fueron al exterior: José Wynne y Andrés Gallo; y quienes siguieron la carrera académica: Christian Volpe, Silvia Marchionni, Malena Monteverde, Fernando Cuenin, Matías Busso. En las memorias del Departamento de Economía de la UNLP correspondientes al período bajo análisis se pueden encontrar las referencias a la actividad académica de la mayoría de ellos. Al menos hasta el año 2014 en que se recogió el testimonio, el GIE se incorporó formalmente a la Dirección Provincial de Estudios Económicos del Ministerio, y su actividad era coordinada por León Salim.

13 La experiencia se extendería hasta el año 2002, en que dio lugar a la empresa de servicios “Abeceb”, dirigida por el Lic. Dante Sica (http://www.abeceb.com/web/home/aboutus [visita 10-03-2016]).

14 "era un centro que estudiaba la economía nacional pero desde la perspectiva de la provincia ... la idea nuestra siempre era cómo mirábamos la economía nacional desde un grupo de economistas que ... tenían raigambre en la provincia de Buenos Aires ... éramos especialistas en finanzas provinciales pero ... nuestra idea era tener injerencia a nivel nacional ... 
en su momento el Centro lo hicimos como una proyección política ..., era desde donde opinar de política económica, y ... ponernos como referencia. Si vos mirás los documentos siempre fuimos muy críticos" (ex funcionario del Ministerio de Economía de la Provincia de Buenos Aires, consultor económico).

15 El Instituto de Desarrollo Económico y Social, fundado en 1958 a instancias de Aldo Ferrer, reconocido economista argentino que en ese entonces era Ministro de Economía de la Provincia de Buenos Aires, significó un espacio académico en el que confluyeron economistas, sociólogos, historiadores y politólogos, que alumbró una de las revistas académicas más prestigiosas en el campo de las ciencias sociales a nivel nacional, la Revista de Desarrollo Económico. Ver http://ide s.org.ar/acerca-del-ides/historia[visitado el 4-05-2016].

16 A diferencia del CEB, el IEFE continúa con sus actividades hasta el momento de producir este trabajo.

17 Los economistas incorporados eran también docentes adjuntos de la carrera de economía de la UNLP, enrolados en las corrientes "heterodoxas", como Demián Panigo, Pablo Pérez y Néstor Félix (Departamento de Economía, Memoria 2007).

18 La revista se publicó con frecuencia bimestral a lo largo del período de gestión de Remes Lenicov (1989-1997). En los siguientes años su continuidad fue más accidentada, registrándose algunos en los que no pudo mantener esa frecuencia, al punto de discontinuarse durante la gestión de Sarghini (1997-2003), para ser retomada en la de Otero (2003-2007), aunque no lograría recuperar el ritmo bimestral de publicación. En total, entre 1989 y 2006 se publicaron 77 números, 48 de los cuales correspondieron a la gestión de Remes Lenicov, 17 a la de Sarghini, y 12 a la de Otero.

19 Hasta el No 18 contienen una "carta al lector" que anunciaba los principales temas tratados.

20 Estas secciones hacían referencia a los tres ejes alrededor de los cuales el Ministerio organizó sus "programas de acción”, descriptos en la Noticias 1 (sep-oct 1989).

21 A modo de ejemplo, en el ya referido $\mathrm{N}^{\circ} 66$ un artículo titulado "La crítica a la ortodoxia neoliberal llega de la mano de Keynes” estaba dedicado a reseñar la intervención de Axel Leijonhufvud, reconocido economista sueco especializado en Keynes, en las VIII Jornadas de Economía Monetaria e Internacional desarrolladas en la Facultad de Ciencias Económicas de la UNLP, "en abierta confrontación con la teoría macroeconómica tradicional ... liderada por Robert Lucas Jr.”, uno de los teóricos neoclásicos más recientes, Premio Nobel en 1995, que contribuyó a renovar esa corriente de pensamiento a partir de la teoría de las expectativas racionales (Noticias de Economía 66: 30). En el Nº68, de octubrenoviembre de 2003, una nota sin firma -presumiblemente preparada por integrantes del GIE- bajo el título de "El fracaso del pensamiento único", realizaba una reseña crítica de la política económica orientada por el denominado "Consenso de Washington", y daba cuenta de la experiencia argentina a partir de la crisis de 2001, que tomaba decidida distancia de las prescripciones asociadas con el mismo.

22 La figura del "barril sin fondo" propuesta por Eugene Bardach (citado en Aguilar Villanueva, 1994: II.3), da cuenta de la tendencia a la utilización indiscriminada de los fondos, sin establecer prioridades claras y sin considerar la generación de una deuda que en algún momento habría que pagar; el supuesto que subyace es el de una oferta ilimitada de recursos, y que "paga la próxima gestión".

23 Desde que se inició y hasta el año 2007 se publicaron 74 números. Desde noviembre de 1994, la publicación tuvo continuidad mensual a lo largo de 1995, 1996 (con excepción de los meses de enero y febrero), 1997 y 1998. En 1999 dejaron de editarse, retomándose en diciembre de ese año. En el año 2000 se publicaron 8 Cuadernos (enero a marzo, julio y agosto, y octubre a diciembre); en el 2001 otros 8 (enero a marzo, abril a junio, octubre y diciembre); en el 2002 sólo 4 (enero, junio y julio, y octubre); en el 2003 se interrumpió nuevamente la publicación, y se editaron sólo 2 números en 2004 (agosto y octubre), 4 en 2005 (abril a junio y agosto), y 2 en 2006 (mayo y junio). La publicación se interrumpió en el año 2007, para ser retomada en julio de 2010.De acuerdo con la información de la página institucional del Ministerio de Economía provincial, la publicación habría quedado nuevamente trunca, ya que el último número informado es el 79, de diciembre de 2011 (http://www.ec.gba.gov.ar/prensa/cuadernos_economia.php [visita 15-04-2016] y http://w ww.ec.gba.gov.ar/areas/estudios_proyecciones/cuadernos_economia.php [visita 21-04-2017]).

24 No existe en nuestro país un registro delas citaciones de trabajos y publicaciones académicas que permitan medir su impacto en términos de circulación dentro de la comunidad.Lo más próximo es reconstruir de manera artesanal cuánto circulan algunos autores. Una búsqueda sencilla por el navegador más utilizado, el Google, permitió comprobar que los Cuadernos son citados en trabajos especializados y ponencias, se utilizan como bibliografía en materias, pero que dicha circulación no alcanza niveles masivos, sino que por el contrario es circunscripta y de carácter "parroquial”.

25 Los Cuadernos 3 "Propuesta para un sistema tributario federal”, 6 "Gasto público provincial y municipal por partido de la Provincia de Buenos Aires”, 8 “Impacto distributivo del gasto público provincial y municipal en la Provincia de Buenos Aires", 14 "Dos problemas de finanzas públicas: El crecimiento del gasto público y las potestades del endeudamiento provincial” y 15 "Estudio sobre finanzas provinciales y el sistema de coparticipación federal de impuestos", recogieron resultados de investigación de equipos de investigación coordinados por Alberto Porto.

26 Los Cuadernos 23 "Estructura y rendimiento de los sistemas tributarios de Argentina y de algunos países seleccionados de América Latina y OECD”, 31 "Lineamientos para una reforma del Sistema tributario argentino", 57 "Instituciones y reforma fiscal federal en la Argentina”, estuvieron a cargo de consultores contratados por el Programa de Apoyo a 
la Reforma Impositiva de la Provincia de Buenos Aires, financiado por el Programa de las Naciones Unidas para el Desarrollo (PNUD) los dos primeros, y de integrantes del GIE el último.

27 Ejemplos de ello son los Cuadernos 47 "La Provincia de Buenos Aires: una mirada a su economía real”, 52 "Crecimiento sostenido y con equidad: el rol del financiamiento", 59 "El dilema de la economía informal: evidencias y políticas”, 61, 64, 66, 69 y 70, dedicados a clusters productivos en la Provincia de Buenos Aires, 67 "Determinantes y obstáculos de la inserción externa de los complejos productivos bonaerenses", 71 "Exportaciones y tipo de cambio real en la Argentina”.

28 Tal como lo demuestran, por ejemplo, los Cuadernos 13 "Niveles de desarrollo económico por provincias: indicadores y evolución intertemporal", 22 "Concentración territorial de la industria argentina", 25 "Empleo y desempleo en la Argentina”, 41 "Evolución regional (por provincias) de la actividad económica en el sector comercio y servicios” y 43 "Análisis de la actividad económica en la Provincia de Buenos Aires".

29 Todo esto se halla más desarrollado en el Capítulo 5 de mi tesis.

30 Se consignan únicamente las que aparecen citadas. 